\title{
Time evolution of Wikipedia network ranking
}

\author{
Young-Ho Eom ${ }^{1}$, Klaus M. Frahm ${ }^{1}$, András Benczúr ${ }^{2}$, and Dima L. Shepelyansky ${ }^{1, a}$ \\ 1 Laboratoire de Physique Théorique du CNRS, IRSAMC, Université de Toulouse, UPS, 31062 Toulouse, France \\ 2 Informatics Laboratory, Institute for Computer Science and Control, Hungarian Academy of Sciences (MTA SZTAKI), \\ Pf. 63, 1518 Budapest, Hungary
}

Received 24 April 2013 / Received in final form 4 September 2013

Published online 2 December 2013 - C EDP Sciences, Società Italiana di Fisica, Springer-Verlag 2013

\begin{abstract}
We study the time evolution of ranking and spectral properties of the Google matrix of English Wikipedia hyperlink network during years 2003-2011. The statistical properties of ranking of Wikipedia articles via PageRank and CheiRank probabilities, as well as the matrix spectrum, are shown to be stabilized for 2007-2011. A special emphasis is done on ranking of Wikipedia personalities and universities. We show that PageRank selection is dominated by politicians while 2DRank, which combines PageRank and CheiRank, gives more accent on personalities of arts. The Wikipedia PageRank of universities recovers $80 \%$ of top universities of Shanghai ranking during the considered time period.
\end{abstract}

\section{Introduction}

At present Wikipedia [1] has become the world largest encyclopedia with open public access to its content. A recent review [2] represents a detailed description of publications and scientific research on information storage at Wikipedia and its classification. Wikipedia contains an enormous amount of information and, in a certain sense, the problem of information arrangement and retrieval from its contain starts to remind similar information problems in the Library of Babel described by Borges [3]. The hyperlinks between Wikipedia articles represent a directed network which reminds a structure of the World Wide Web (WWW). Hence, the mathematical tools developed for WWW search engines, based on the Markov chains [4], Perron-Frobenius operators [5] and the PageRank algorithm of the corresponding Google matrix $[6,7]$, give solid mathematical grounds for analysis of information flow on the Wikipedia network. In this work we perform the Google matrix analysis of Wikipedia network of English articles extending the results presented in [8-11]. The main new element of this work is the study of time evolution of Wikipedia network during the years 2003 to 2011. We analyze how the ranking of Wikipedia articles and the spectrum of the Google matrix $G$ of Wikipedia are changed during this period.

The directed network of Wikipedia articles is constructed in a standard way: a directed link is formed from an article $j$ to an article $i$ when $j$ quotes $i$ and an element $A_{i j}$ of the adjacency matrix is taken to be unity when there is such a link and zero in absence of link. The

\footnotetext{
a e-mail: dima@irsamc.ups-tlse.fr
}

columns with only zero elements (dangling nodes) are replaced by columns with $1 / N$ with $N$ being the matrix size. The elements of other columns are renormalized in such a way that their sum becomes equal to unity $\left(\sum_{i} S_{i j}=1\right.$, $\left.S_{i j}=A_{i j} / \sum_{i} A_{i j}\right)$. Thus we obtain the matrix $S_{i j}$ of Markov transitions. Then the Google matrix of the network takes the form $[6,7]$ :

$$
G_{i j}=\alpha S_{i j}+(1-\alpha) / N
$$

The damping parameter $\alpha$ in the WWW context describes the probability $(1-\alpha)$ to jump to any node for a random surfer. For WWW the Google search engine uses $\alpha \approx 0.85$ [7]. The matrix $G$ belongs to the class of PerronFrobenius operators $[5,7]$, its largest eigenvalue is $\lambda=1$ and other eigenvalues have $|\lambda| \leq \alpha$. The right eigenvector at $\lambda=1$, which is called the PageRank, has real nonnegative elements $P(i)$ and gives a probability $P(i)$ to find a random surfer at site $i$. It is possible to rank all nodes in a decreasing order of PageRank probability $P(K(i))$ so that the PageRank index $K(i)$ counts all $N$ nodes $i$ according their ranking, placing the most popular articles or nodes at the top values $K=1,2,3 \ldots$

Due to the gap $1-\alpha \approx 0.15$ between the largest eigenvalue $\lambda=1$ and other eigenvalues the PageRank algorithm permits an efficient and simple determination of the PageRank by the power iteration method [7]. It is also possible to use the powerful Arnoldi method [12-14] to compute efficiently the eigenspectrum $\lambda_{i}$ of the Google matrix:

$$
\sum_{k=1}^{N} G_{j k} \psi_{i}(k)=\lambda_{i} \psi_{i}(j)
$$


Table 1. Parameters of all Wikipedia networks at different years considered in the paper; set 2009 corresponds to December 2009, set 200908 to August 2009.

\begin{tabular}{lccc}
\hline & $N$ & $N_{\ell}$ & $n_{A}$ \\
\hline 2003 & 455436 & 2033173 & 6000 \\
2005 & 1635882 & 11569195 & 6000 \\
2007 & 2902764 & 34776800 & 6000 \\
2009 & 3484341 & 52846242 & 6000 \\
200908 & 3282257 & 71012307 & 6000 \\
2011 & 3721339 & 66454329 & 6000 \\
\hline
\end{tabular}

The Arnoldi method allows to find a several thousands of eigenvalues $\lambda_{i}$ with maximal $|\lambda|$ for a matrix size $N$ as large as a few tens of millions $[10,11,14,15]$. Usually, at $\alpha=1$ the largest eigenvalue $\lambda=1$ is highly degenerate [15] due to many invariant subspaces which define many independent Perron-Frobenius operators providing (at least) one eigenvalue $\lambda=1$.

In addition to a given directed network $A_{i j}$ it is useful to analyze an inverse network with inverted direction of links with elements of adjacency matrix $A_{i j} \rightarrow A_{j i}$. The Google matrix $G^{*}$ of the inverse network is then constructed via corresponding matrix $S^{*}$ according to the relations (1) using the same value of $\alpha$ as for the $G$ matrix. This time inversion approach was used in $[16,17]$ but the statistical properties and correlations between direct and inversed ranking were not analyzed there. In [18], on an example of the Linux Kernel network, it was shown that this approach allows to obtain an additional interesting characterization of information flow on directed networks. Indeed, the right eigenvector of $G^{*}$ at eigenvalue $\lambda=1$ gives a probability $P^{*}(i)$, called CheiRank vector [8]. It determines a complementary rank index $K^{*}(i)$ of network nodes in a decreasing order of probability $P^{*}\left(K^{*}(i)\right)[8-10,18]$. It is known that the PageRank probability is proportional to the number of ingoing links characterizing how popular or known is a given node. In a similar way the CheiRank probability is proportional to the number of outgoing links highlighting the node communicativity (see e.g. [7-9,19-21]). The statistical properties of distribution of indexes $K(i), K^{*}(i)$ on the PageRankCheiRank plane are described in [9].

In this work we apply the above mathematical methods to the analysis of time evolution of Wikipedia network ranking using English Wikipedia snapshots dated by December 31 of years 2003, 2005, 2007, 2009, 2011. In addition we use the snapshot of August 2009 (200908) analyzed in [8]. The parameters of networks with the number of articles (nodes) $N$, number of links $N_{\ell}$ and other information are given in Tables 1 and 2 with the description of notations given in Appendix.

The paper is composed as following: the statistical properties of PageRank and CheiRank are analyzed in Section 2, ranking of Wikipedia personalities and universities are considered in Sections 3, 4 respectively, the properties of spectrum of Google matrix are considered in Section 5, the discussion of the results is presented in Section 6, Appendix gives network parameters.
Table 2. $G$ and $G^{*}$ eigespectrum parameters for all Wikipedia networks, year marks spectrum of $G$, year with star marks spectrum of $G^{*}$.

\begin{tabular}{lccccc}
\hline & $N_{s}$ & $N_{d}$ & $d_{\max }$ & $N_{\text {circ. }}$ & $N_{1}$ \\
\hline 2003 & 15 & 7 & 3 & 11 & 7 \\
$2003^{*}$ & 940 & 162 & 60 & 265 & 163 \\
2005 & 152 & 97 & 4 & 121 & 97 \\
$2005^{*}$ & 5966 & 1455 & 1997 & 2205 & 1458 \\
2007 & 261 & 150 & 6 & 209 & 150 \\
$2007^{*}$ & 10234 & 3557 & 605 & 5858 & 3569 \\
2009 & 285 & 121 & 8 & 205 & 121 \\
$2009^{*}$ & 11423 & 4205 & 134 & 7646 & 4221 \\
200908 & 515 & 255 & 11 & 381 & 255 \\
$200908^{*}$ & 21198 & 5355 & 717 & 8968 & 5365 \\
2011 & 323 & 131 & 8 & 222 & 131 \\
$2011^{*}$ & 14500 & 4637 & 1323 & 8591 & 4673 \\
\hline
\end{tabular}
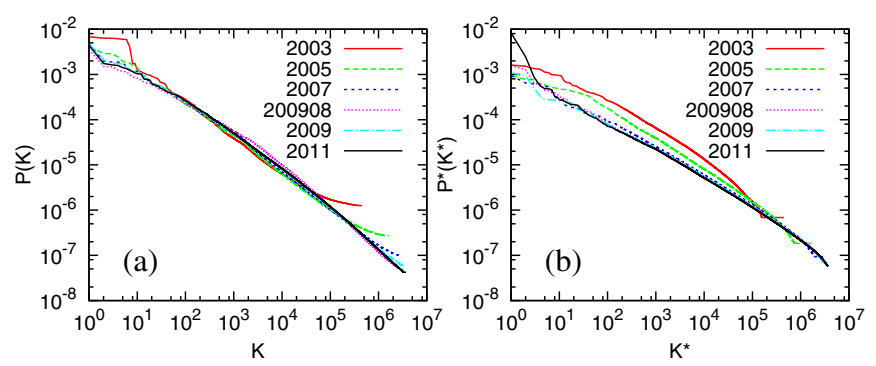

Fig. 1. PageRank probability $P(K)$ (left panel) and CheiRank probability $P^{*}\left(K^{*}\right)$ (right panel) are shown as a function of the corresponding rank indexes $K$ and $K^{*}$ for English Wikipedia articles at years 2003, 2005, 2007, 200908, 2009, 2011; here the damping factor is $\alpha=0.85$.

\section{CheiRank versus PageRank}

The dependencies of PageRank and CheiRank probabilities $P(K)$ and $P^{*}\left(K^{*}\right)$ on their indexes $K, K^{*}$ at different years are shown in Figure 1. The top positions of $K$ are occupied by countries starting from United States while at the top positions of $K^{*}$ we find various lists (e.g. geographical names, prime ministers etc.; in 2011 we have appearance of lists of lists). Indeed, the countries accumulate links from all types of human activities and nature, that make them the most popular Wikipedia articles, while lists have the largest number of outgoing links making them the most communicative articles.

The data of Figure 1 show that the global behavior of $P(K)$ remains stable from 2007 to 2011 . Indeed, the decay of probability curves $P(K)$ is very similar and 4 curves are practically overlapped in $K<10^{6}$. Also the probability decay $P^{*}\left(K^{*}\right)$ is described by curves been very close to each other for the time interval 2007-2009 while at 2011 we see the appearance of peak at $1 \leq K^{*}<10$. This peak is related to introduction of lists of lists which were absent at earlier years. At the same time the behavior of $P^{*}\left(K^{*}\right)$ in the range $10 \leq K^{*} \leq 10^{6}$ remains stable for $2007-2011$. Indeed, we see that the probability curves are very close to each other as it is well visible in Figure 1. However, for 

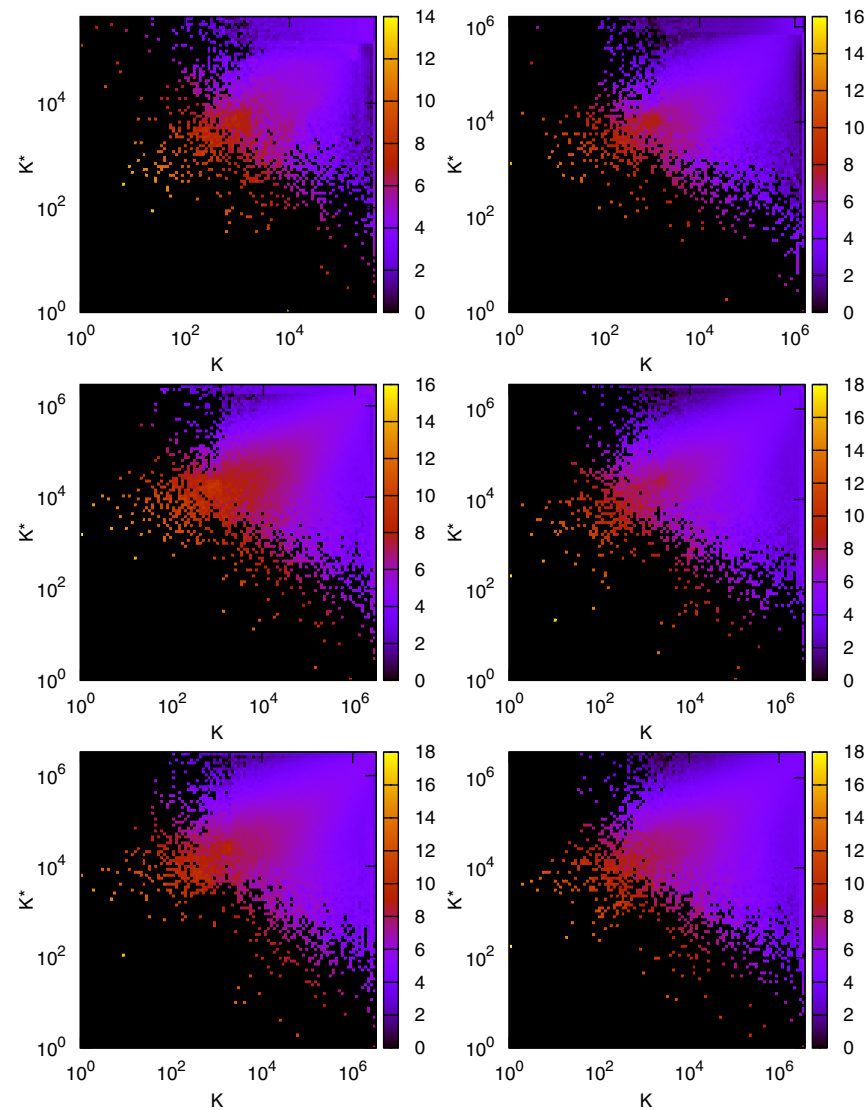

Fig. 2. Density of Wikipedia articles in the CheiRank versus PageRank plane at different years. Color is proportional to logarithm of density changing from minimal nonzero density (dark) to maximal one (white), zero density is shown by black (distribution is computed for $100 \times 100$ cells equidistant in logarithmic scale; bar shows color variation of natural logarithm of density); left column panels are for years 2003, 2007, 200908 and right column panels are for 2005, 2009, 2011 (from top to bottom).

a quantitative analysis one needs to consider overlap of articles in the top ranking at different years. We discuss this point below.

Each article $i$ has its PageRank and CheiRank indexes $K(i), K^{*}(i)$ so that all articles are distributed on twodimensional plane of PageRank-CheiRank indexes. Following $[8,9]$, we present the density of articles in the 2D plane $\left(K, K^{*}\right)$ in Figure 2. The density is computed for $100 \times 100$ logarithmically equidistant cells which cover the whole plane $\left(K, K^{*}\right)$ for each year. Qualitatively we find that the density distribution is globally stable for years 2007-2011 even if definitely there are articles which change their location in 2D plane. For example, we see an appearance of a mountain like ridge of probability located approximately along a line $\ln K^{*} \approx \ln K+4.6$ that indicates the presence of correlation between $P(K(i))$ and $P^{*}\left(K^{*}(i)\right)$. Also the form of density distributions looks to be similar at all years studied even if individual articles change their positions.
Following $[8,9,18]$, we characterize the interdependence of PageRank and CheiRank vectors by the correlator

$$
\kappa=N \sum_{i=1}^{N} P(K(i)) P^{*}\left(K^{*}(i)\right)-1 .
$$

We find the following values of the correlator at various time slots: $\kappa=2.837$ (2003), $3.894(2005), 4.121$ (2007), 4.084 (200908), 6.629 (2009), 5.391 (2011). During that period the size of the network increased almost by 10 times while $\kappa$ increased less than 2 times. The root mean square variation around the average value $\kappa=4.49$ is relatively modest being $\delta \kappa=1.2$. The stability of $\kappa$ is especially visible in comparison with other networks. Indeed, for the network of University of Cambridge we have $\kappa=1.71$ in 2006 and 30 in 2011 [9]. This confirms the stability of the correlator $\kappa$ during the time evolution of the Wikipedia network.

To analyze the stability of ranking in a more quantitative manner, we determine the number of the same overlapping articles at different years among the top 100 articles in PageRank, 2DRank and CheiRank. The dependence of this overlap characteristic $N_{\text {ovlap }}$ on different years is shown in Figure 3. For PageRank we have the lowest value $N_{\text {ovlap }} \approx 40$ and for the time period 2007-2011 we have this value mainly in the range 60-80 confirming the stability of top 100 articles of PageRank. For 2DRank we have smaller values of $N_{\text {ovlap }}$ which are located mainly in the range 30-50 for the period 2007-2011 with overlap drop to 10 between 2003 and 2011. For CheiRank we find approximately the same of overlap as for 2DRank for years 2007-2011. However, e.g. for years 2003 vs. 2011 the overlap for CheiRank drops significantly down to the minimal value $N_{\text {ovlap }}=2$. We attribute this to significant fluctuations of top 100 CheiRank probabilities especially visible in Figure 1 for years 2003, 2005. The significant values of overlap parameter $N_{\text {ovlap }}$ for years $2007-2011$ indicate the stabilization of rank distributions in this period.

In the next two sections we analyze the time variation of ranking of personalities and universities.

\section{Ranking of personalities}

To analyze the time evolution of ranking of Wikipedia personalities (persons or humans) we chose the top 100 persons appearing in the ranking list of Wikipedia 200908 given in [8] in order of PageRank, CheiRank and 2DRank. We remind that 2 DRank $K_{2}$ is obtained by counting nodes in order of their appearance on ribs of squares in $\left(K, K^{*}\right)$ plane with their size growing from $K=1$ to $K=N$ [8].

The distributions of personalities in PageRankCheiRank plane is shown at various time slots in Figure 4. There are visible fluctuations of distribution of nodes for years 2003 and 2005 when the Wikipedia size has rapid growth (see, e.g., Figs. 4a and 4c). For other years 2007-2011 the distribution of top 100 nodes of personalities of PageRank and 2DRank is more compact even if individual nodes change their rank positions in 


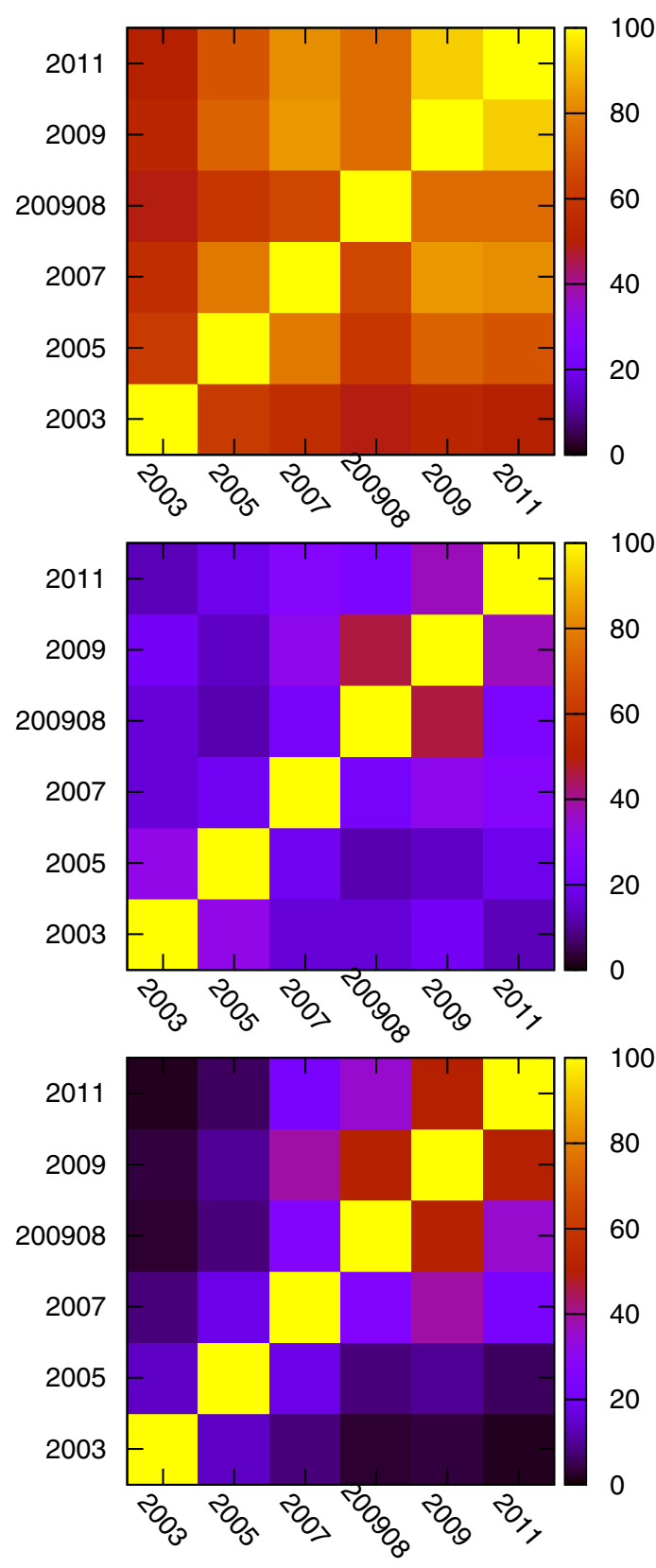

Fig. 3. Number of the same overlapped articles between top 100 Wikipedia articles at different years for ranking by PageRank (top panel), 2DRank (middle panel) and CheiRank (bottom panel).

$\left(K, K^{*}\right)$ plane: in these years the points form one compact cloud (see Figs. 4a and 4c). For top 100 personalities of CheiRank the fluctuations remain strong during all years (Fig. 4b). Indeed, the number of outgoing links is more easy to be modified by authors writing a given article, while a modification of ingoing links depends on authors of other articles.

In Figure 4, we also show the distribution of top 100 personalities from Hart's book [22] (the list of names is also available at the web page [8]). This distribution also remains stable in years 2007-2011. It is interesting to note
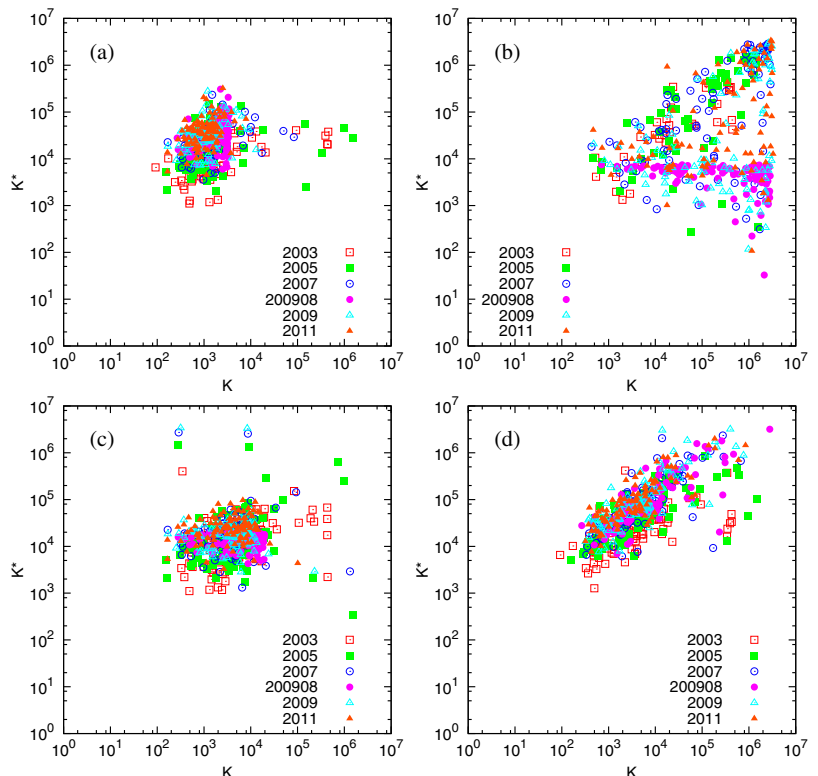

Fig. 4. Change of locations of top-rank persons of Wikipedia in $K-K^{*}$ plane. Each list of top ranks is determined by data of top 100 personalities of time slot 200908 in corresponding rank. Data sets are shown for (a) PageRank, (b) CheiRank, (c) 2DRank, (d) rank from Hart [22].

that while top PageRank and 2DRank nodes form a kind of droplet in $\left(K, K^{*}\right)$ plane, the distribution of Hart's personalities approximately follows the ridge along the line $\ln K^{*} \approx \ln K+4.6$.

The time evolution of top 10 personalities of slot 200908 is shown in Figure 5 for PageRank and 2DRank. For PageRank the main part of personalities keeps their rank position in time, e.g. G.W. Bush remains at firstsecond position. B. Obama significantly improves his ranking as a result of president elections. There are strong variations for Elizabeth II which we relate to modification of article name during the considered time interval. We also see a steady improvement of ranking of C. Linnaeus that we attribute to a growth of descriptions of various botanic, insect and animal species which quote C. Linnaeus. For 2DRank we observe stronger variations of $K_{2}$ index with time. Such a politician as R. Nixon has increasing $K_{2}$ index with time since the period of his presidency is finished more and more years ago and events linked to his political activity, e.g. like Watergate scandal, have lower and lower echo with time. At the same time such representatives of arts as M. Jackson, F. Sinatra, and S. King remain at approximately constant level of $K_{2}$ or even improve their ranking.

We note that in Figure 5 the dispersion of points increases in both directions of time from the slot 200908. This happens because top 10 persons are taken at this moment of time and thus as for any diffusion process the dispersion grows forward and backward in time. Thus we checked that if we take top 10 persons in December 2009 then the dispersion increases in both directions of time from this point. 

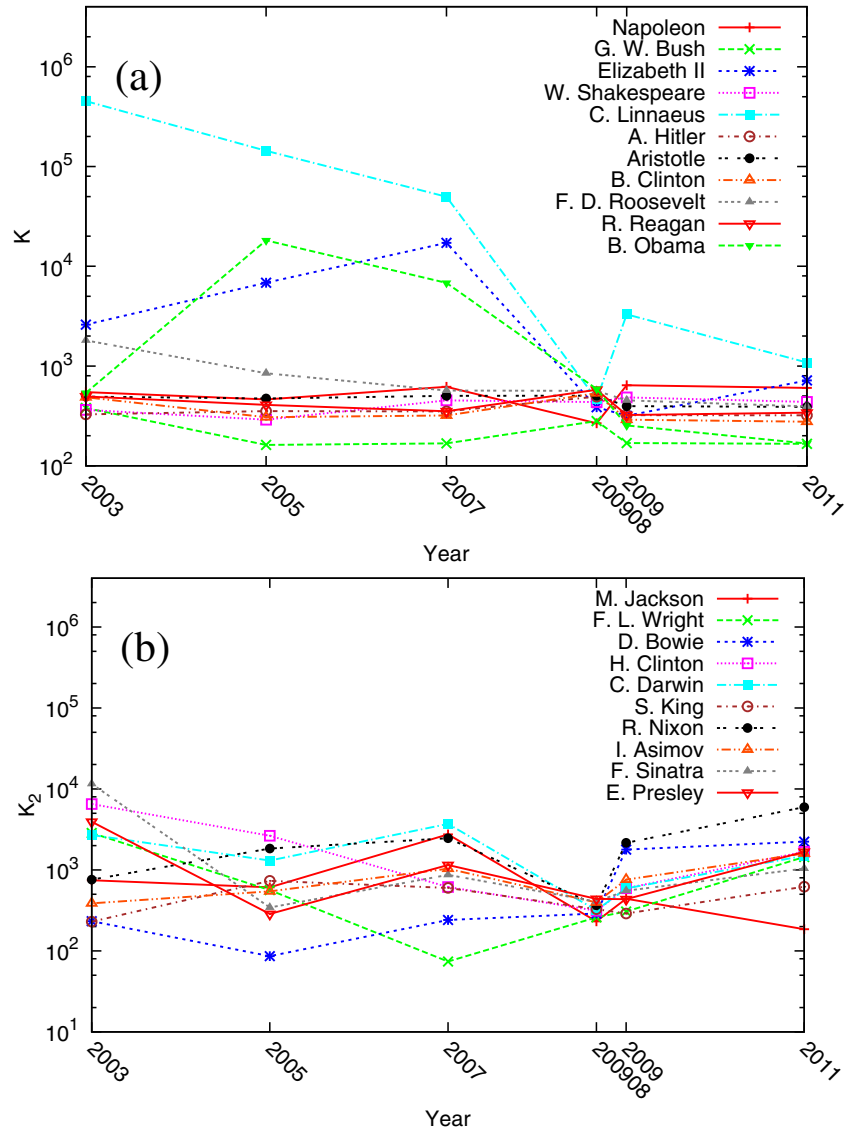

Fig. 5. Time evolution of top 10 personalities of year 200908 in indexes of PageRank $K$ (a) and 2DRank $K_{2}$ (b); B. Obama is added in panel (a).

In [8] it was pointed out that the top personalities of PageRank are dominated by politicians while for 2DRank the dominant component of human activity is represented by artists. We analyze the time evolution of the distribution of top 30 personalities over 6 categories of human activity (politics, arts, science, religion, sport, etc. (or others)). We attribute a personality to an activity following the description of Wikipedia article about this person (presidents, kings, imperators belong to politics, artists, singers, composers, painters belong to arts scientists and philosophers to science, priests and popes to religion, sportsmen to sport, etc. includes all other activities not listed above). In fact, the category etc. contains only C. Columbus. The results are presented in Figure 6. They clearly show that the PageRank personalities are dominated by politicians whose percentage increases with time, while the percent of arts decreases. For 2DRank we see that the arts are dominant even if their percentage decreases with time. We also see the appearance of sport which is absent in PageRank. The mechanism of the qualitative ranking differences between two ranks is related to the fact that 2DRank takes into account via CheiRank a contribution of outgoing links. Due to that singers, actors, sportsmen improve their CheiRank and 2DRrank positions since articles about them contain various music

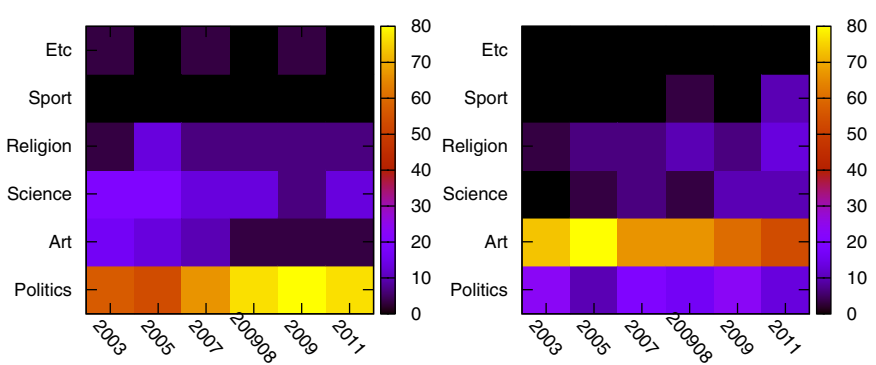

Fig. 6. Left panel: distribution of top 30 PageRank personalities over 6 activity categories at various years of Wikipedia. Right panel: distribution of top 30 2DRank personalities over the same activity categories at same years. Categories are politics, art, science, religion, sport, etc. (other). Color shows the number of personalities for each activity expressed in percents.

albums, movies and sport competitions with many outgoing links. Due to that the component of arts gets higher positions in 2DRank in contrast to politics dominance in PageRank. Thus the two-dimensional ranking on PageRank-CheiRank plane allows to select qualities of nodes according to their popularity and communicativity.

\section{Ranking of universities}

The local ranking of top 100 universities is shown in Figure 7 for years 2003, 2005, 2007 and in Figure 8 for 2009, 200908, 2011. The local ranking is obtained by selecting top 100 universities appearing in PageRank list so that they get their university ranking $K$ from 1 to 100. The same procedure is done for CheiRank list of universities obtaining their local CheiRank index $K^{*}$ from 1 to 100 . Those universities which enter inside $100 \times 100$ square on the local index plane $\left(K, K^{*}\right)$ are shown in Figures 7 and 8 .

The data show that the top PageRank universities are rather stable in time, e.g. Harvard is always on the first top position, Columbia at the second position and Yale is the third for the majority of years. Also there is a relatively small number of intersection of curves of $K$ with years. At the same time the positions in $K_{2}$ and $K^{*}$ are strongly changing in time. To understand the origin of this variations in CheiRank we consider the case of U. Cambridge. Its Wikipedia article in 2003 is rather short but it contains the list of all 31 colleges with direct links to their corresponding articles. This leads to a high position of U. Cambridge with university $K^{*}=4$ in 2003 (Fig. 9). However, with time the direct links remain only to about 10 colleges while the whole number of colleges are presented by a list of names without links. This leads to a significant increase of index up to $K^{*} \approx 40$ at Dec. 2009. However, at Dec. 2011 U. Cambridge again improves significantly its CheiRank obtaining $K^{*}=2$. The main reason of that is the appearance of section of "Notable alumni and academics" which provides direct links to articles about outstanding scientists studied and/or worked at U. Cambridge that leads to second position at $K^{*}=2$ among all universities. We note that in 2011 the top CheiRank University is George Mason university with university $K^{*}=1$. The main reason of this 

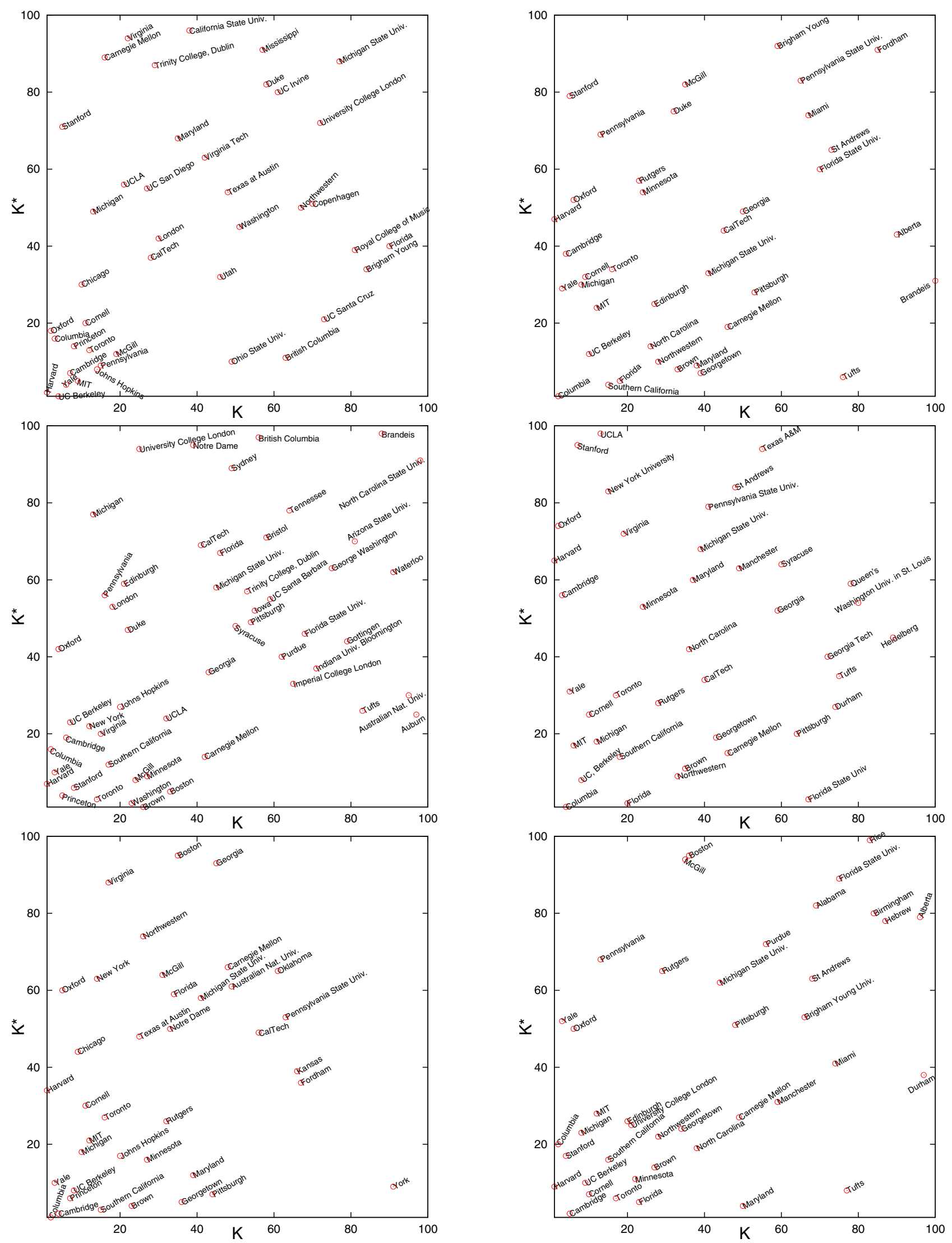

Fig. 7. University of Wikipedia articles in the local CheiRank versus PageRank plane at different years; panels are for years 2003, 2005, 2007 (from top to bottom).

Fig. 8. Same as in Figure 7 for years 2009, 200908, 2011 (from top to bottom). 

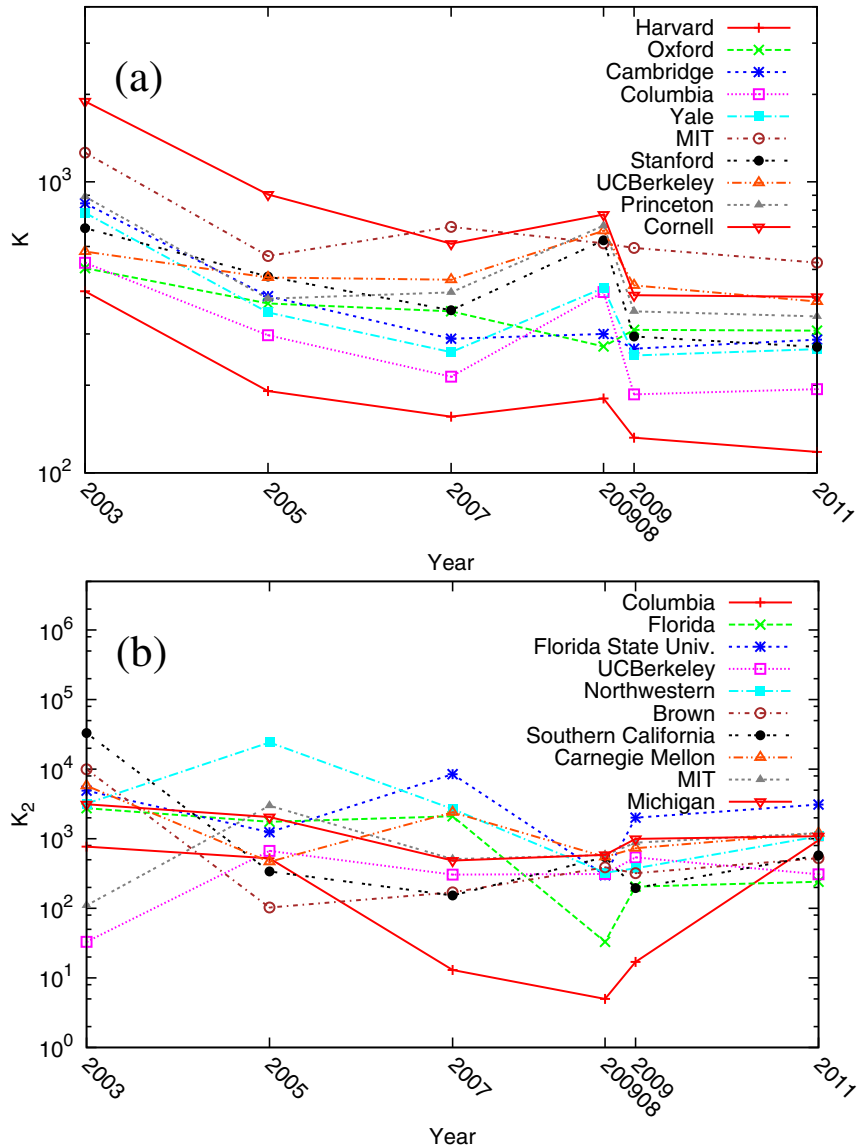

Fig. 9. Time evolution of global ranking of top 10 universities of year 200908 in indexes of PageRank $K$ (a) and 2DRank $K_{2}(\mathrm{~b})$.

high ranking is the presence of detailed lists of alumni in politics, media, sport with direct links to articles about corresponding personalities (including former director of CIA). These two examples show that the links, kept by a university with a large number of its alumni, significantly increase CheiRank position of university. We note that colleges specialized in arts, religion, politics usually preserve more links with their alumni as also was pointed in $[8]$.

The time evolution of global ranking of top 10 universities of year 200908 for PageRank and 2DRank is shown in Figure 9. The results show the stability of PageRank order with a clear tendency of top universities (e.g. Harvard) to go with time to higher and higher top positions of $K$. Thus for Harvard the global value of $K$ changes from $K \approx 300$ in 2003 to $K \approx 100$ in 2011, while the whole size $N$ of the Wikipedia network increases almost by a factor 10 during this time interval. Since Wikipedia ranks all human knowledge, the stable improvement of PageRank indexes of universities reflects the global growing importance of universities in the world of human activity and knowledge.

The time evolution of top 10 universities of year 200908 in 2DRank remains on average approximately at a constant level $K_{2} \approx$ const. in time (without the above global improvement visible for for PageRank), it also shows more interchanges of ranking order comparing to PageRank case. We think that an example of U. Cambridge considered above explains the main reasons of these fluctuations. In view of 10 times increase of the whole network size during the period 2003-2011 the average stability of 2DRank of universities also confirms the significant importance of their place in human activity.

Finally we compare the Wikipedia ranking of universities in their local PageRank index $K$ with those of Shanghai university ranking [23]. In the top 10 of Shanghai university rank the Wikipedia PageRank recovers 9 (2003), 9 (2005), 8 (2007), 7 (2009), 7 (2011). Thus on average the Wikipedia PageRanking of universities recovers $80 \%$ of top universities of Shanghai ranking during the considered time period. This shows that the Wikipedia ranking of universities gives the results being rather similar to Shanghai ranking performed on the basis of other selection criteria. A small decrease of overlap with time can be attributed to earlier launched activity of leading universities on Wikipedia.

\section{Google matrix spectrum}

Finally we discuss the time evolution of the spectrum of Wikipedia Google matrix taken at $\alpha=1$. We perform the numerical diagonalization based on the Arnoldi method [12,13] using the additional improvements described in $[14,15]$ with the Arnold dimension $n_{A}=6000$. The Google matrix is reduced to the form

$$
S=\left(\begin{array}{cc}
S_{s s} & S_{s c} \\
0 & S_{c c}
\end{array}\right)
$$

where $S_{s s}$ describes disjoint subspaces $V_{j}$ of dimension $d_{j}$ invariant by applications of $S ; S_{c c}$ depicts the remaining part of nodes forming the wholly connected core space. We note that $S_{s s}$ is by itself composed of many small diagonal blocks for each invariant subspace and hence those eigenvalues can be efficiently obtained by direct ("exact") numerical diagonalization. The total subspace size $N_{s}$, the number of independent subspaces $N_{d}$, the maximal subspace dimension $d_{\max }$ and the number $N_{1}$ of $S$ eigenvalues with $\lambda=1$ are given in Table 2 (see also Appendix). The spectrum and eigenstates of the core space $S_{c c}$ are determined by the Arnoldi method with Arnoldi dimension $n_{A}$ giving the eigenvalues $\lambda_{i}$ of $S_{c c}$ with largest modulus. Due to the finite value of $n_{A}$ available for numerical simulations eigenvalues with small $\left|\lambda_{i}\right|$ are not computed that leaves an empty space in the complex plane $\lambda$ (see discussions in $[10,11])$. Here we restrict ourselves to the statistical analysis of the spectrum $\lambda_{i}$. The analysis of eigenstates $\psi_{i}\left(G \psi_{i}=\lambda_{i} \psi_{i}\right)$, which has been done in [11] for the slot 200908 , is left for future studies for other time slots.

The spectrum for all Wikipedia time slots is shown in Figure 10 for $G$ and in Figure 11 for $G^{*}$. We see that the spectrum remains stable for the period 2007-2011 even if there is a small difference of slot 200908 due to a slightly different cleaning link procedure (see Appendix). For the spectrum of $G^{*}$ in 2007-2011 we observe a well 

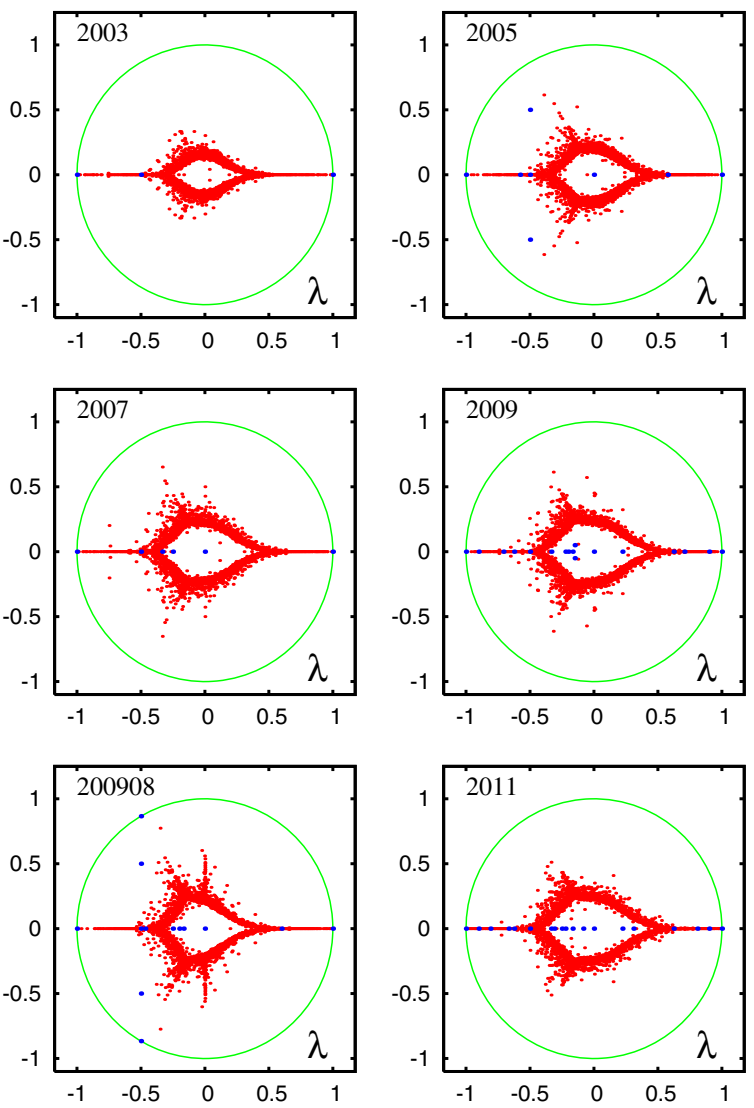

Fig. 10. Spectrum of eigenvalues $\lambda$ of the Google matrix $G$ of Wikipedia at different years shown at $\alpha=1$. Red dots are core space eigenvalues, blue dots are subspace eigenvalues and the full green curve shows the unit circle. The core space eigenvalues were calculated by the projected Arnoldi method with Arnoldi dimensions $n_{A}=6000$.

pronounced star structure which can be recognized as a composition of triplet and quadruplet leaves (triangle and cross). This structure is very similar to those found in random unistochastic and orthostochastic matrices of size $N=3$ and 4 [24] (see Fig. 4 therein). This fact has been pointed in [11] for the slot 200908. Now we see that this is a generic phenomenon which remains stable in time. This indicates that there are dominant groups of 3-4 nodes which have structure similar to random unistochastic or orthostochastic matrices with strong ties between 3-4 nodes and various random permutations with random hidden complex phases. The spectral star structure is significantly more pronounce for the case of $G^{*}$ matrix. We attribute this to more significant fluctuations of outgoing links that probably makes sectors of $G^{*}$ to be more similar to elements of unistochastic matrices. A further detailed analysis will be useful to understand this star structure and its links with various communities inside Wikipedia.

As it is shown in [11] the eigenstates of $G$ and $G^{*}$ select certain well-defined communities of the Wikipedia network. Such an eigenvector detection of the communities provides a new method of communities detection in addition to more standard methods developed in network science and described in [25]. However, the analysis of
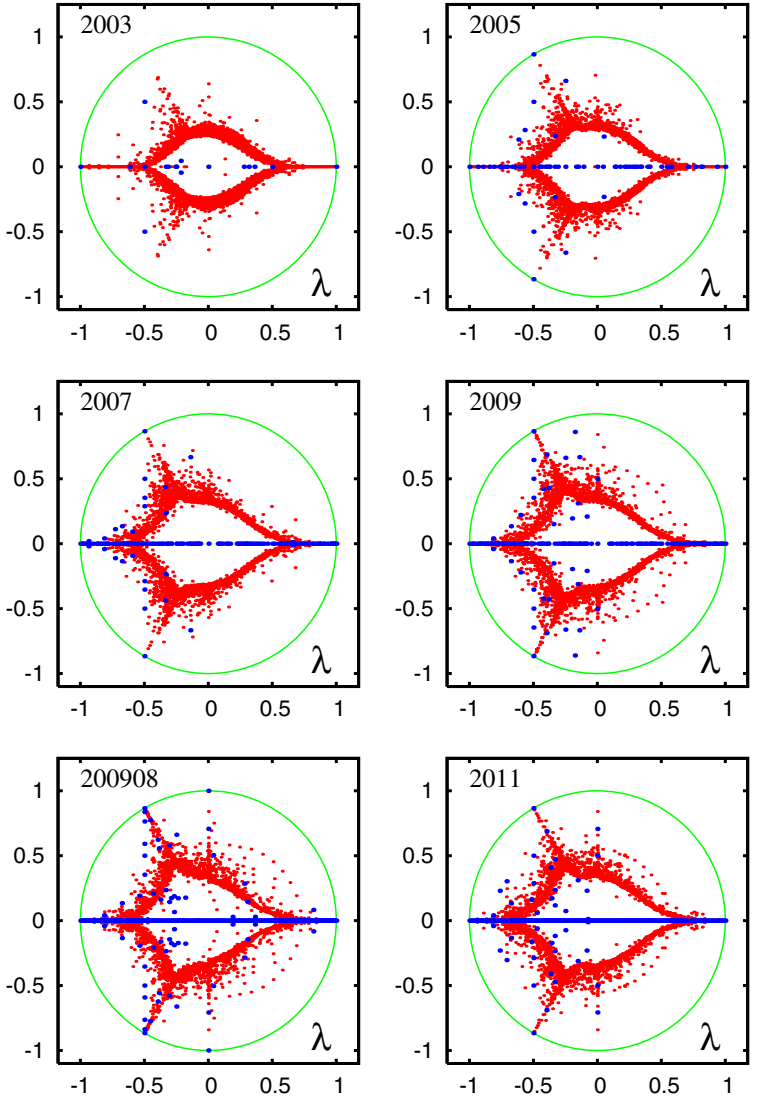

Fig. 11. Same as in Figure 10 but for the spectrum of matrix $G^{*}$.

eigenvectors represents a separate detailed research and in this work we restrict ourselves to PageRank and CheiRank vectors.

Finally we note that the fraction of isolated subspaces is very small for $G$ matrix. It is increased approximately by a factor 10 for $G^{*}$ but still it remains very small compared to the networks of UK universities analyzed in [15]. This fact reflects a strong connectivity of network of Wikipedia articles.

\section{Discussion}

In this work we analyzed the time evolution of ranking of network of English Wikipedia articles. Our study demonstrates the stability of such statistical properties as PageRank and CheiRank probabilities, the article density distribution in PageRank-CheiRank plane during the period 2007-2011. The analysis of human activities in different categories shows that PageRank gives main accent to politics while the combined 2DRank gives more importance to arts. We find that with time the number of politicians in the top positions increases. Our analysis of ranking of universities shows that on average the global ranking of top universities goes to higher and higher positions. This clearly marks the growing importance of universities for the whole range of human activities and knowledge. We find that Wikipedia PageRank recovers $70-80 \%$ of top 10 
universities from Shanghai ranking [23]. This confirms the reliability of Wikipedia ranking.

We also find that the spectral structure of the Wikipedia Google matrix remains stable during the time period 2007-2011 and show that its arrow star structure reflects certain features of small size unistochastic matrices.

Our research presented here is supported in part by the EC FET Open project "New tools and algorithms for directed network analysis" (NADINE No. 288956). This work was granted access to the HPC resources of CALMIP (Toulouse) under the allocations 2012-P0110, 2013-P0110. We also acknowledge the France-Armenia collaboration Grant CNRS/SCS No. 24943 (IE-017) on "Classical and quantum chaos".

\section{Appendix}

The tables with all network parameters used in this work are given Tables 1 and 2. The notations used in the tables are: $N$ is network size, $N_{\ell}$ is the number of links, $n_{A}$ is the Arnoldi dimension used for the Arnoldi method for the core space eigenvalues, $N_{d}$ is the number of invariant subspaces, $d_{\max }$ gives a maximal subspace dimension, $N_{\text {circ. }}$ notes number of eigenvalues on the unit circle with $\left|\lambda_{i}\right|=1, N_{1}$ notes number of unit eigenvalues with $\lambda_{i}=1$. We remark that $N_{s} \geq N_{\text {circ. }} \geq N_{1} \geq N_{d}$ and $N_{s} \geq d_{\text {max }}$. The data for $G$ are marked by the corresponding year of the time slot, the data for $G^{*}$ are marked by the year with a star. Links cleaning procedure eliminates all redirects for sets of 2003, 2005, 2007, 2009, 2011 (nodes with one outgoing link are eliminated; thus practically all redirects are eliminated, we do no relink articles via redirects). Also all articles which titles have only numbers or/and only special symbols have been eliminated. The set 200908 is taken from [8] where the cleaning procedure was slightly different: all nodes with one outgoing link were eliminated but no special cleaning on article titles with numbers was affected. This is probably the reason why $N_{l}$ of 200908 is larger than its values in Dec. 2009 and 2011. All data sets and high resolution figures are available at the web page [26].

\section{References}

1. Wikipedia, en.wikipedia.org/wiki/Wikipedia

2. F.A. Nielsen, Wikipedia research and tools: review and comments, available at SSRN: dx.doi.org/10.2139/ssrn.2129874 (2012)
3. J.L. Borges, The Library of Babel in Ficciones (Grove Press, New York, 1962)

4. A.A. Markov, Rasprostranenie zakona bol'shih chisel na velichiny, zavisyaschie drug ot druga, Izvestiya Fizikomatematicheskogo obschestva pri Kazanskom universitete, 2-ya seriya 15, 135 (1906) (in Russian) [English trans.: Extension of the limit theorems of probability theory to a sum of variables connected in a chain reprinted in Appendix B of: R.A. Howard Dynamic Probabilistic Systems, volume 1: Markov models (Dover Publ., 2007)]

5. M. Brin, G. Stuck, Introduction to dynamical systems (Cambridge University Press, Cambridge, 2002)

6. S. Brin, L. Page, Computer Networks and ISDN Systems 30, 107 (1998)

7. A.M. Langville, C.D. Meyer, Google's PageRank and Beyond: The Science of Search Engine Rankings (Princeton University Press, Princeton, 2006)

8. A.O. Zhirov, O.V. Zhirov, D.L. Shepelyansky, Eur. Phys. J. B 77, 523 (2010)

9. L. Ermann, A.D. Chepelianskii, D.L. Shepelyansky, J. Phys. A 45, 275101 (2012)

10. K.M. Frahm, D.L. Shepelyansky, Eur. Phys. J. B 85, 355 (2012)

11. L. Ermann, K.M. Frahm, D.L. Shepelyansky, Eur. Phys. J. B 86, 193 (2013)

12. G.W. Stewart, Matrix Algorithms Eigensystems (SIAM, Philadelphia, 2001), Vol. II

13. G.H. Golub, C. Greif, BIT Num. Math. 46, 759 (2006)

14. K.M. Frahm, D.L. Shepelyansky, Eur. Phys. J. B 76, 57 (2010)

15. K.M. Frahm, B. Georgeot, D.L. Shepelyansky, J. Phys. A 44, 465101 (2011)

16. D. Fogaras, Lect. Notes Comput. Sci. 2877, 65 (2003)

17. V. Hrisitidis, H. Hwang, Y. Papakonstantino, ACM Trans. Database Syst. 33, 1 (2008)

18. A.D. Chepelianskii, Towards physical laws for software architecture (2010), arXiv: 1003.5455 [cs.SE], www. quantware.ups-tlse.fr/QWLIB/linuxnetwork/

19. D. Donato, L. Laura, S. Leonardi, S. Millozzi, Eur. Phys. J. B 38, 239 (2004)

20. G. Pandurangan, P. Raghavan, E. Upfal, Internet Math. 3, 1 (2005)

21. N. Litvak, W.R.W. Scheinhardt, Y. Volkovich, Lect. Notes Comput. Sci. 4936, 72 (2008)

22. M.H. Hart, The 100: ranking of the most influential persons in history (Citadel Press, New York, 1992)

23. www. shanghairanking. com/

24. K. Zyczkowski, M. Kus, W. Slomczynski, H.-J. Sommers, J. Phys. A 36, 3425 (2003)

25. S. Fortunato, Phys. Rep. 486, 75 (2010)

26. www. quantware.ups-tlse.fr/QWLIB/

wikirankevolution/ 